\title{
The transition to online teaching during the COVID-19 pandemic at a regional, rural university: The experience of learning assistants
}

\author{
Melanie Fields, ${ }^{1}$ Bahar Modir, ${ }^{2}$ William G. Newton, ${ }^{2}$ Robynne M. Lock, ${ }^{2}$ and J. Clay Stanfield ${ }^{2}$ \\ ${ }^{1}$ Department of Curriculum \& Instruction, Texas A\&M University-Commerce, Commerce, TX, 75429, USA \\ ${ }^{2}$ Department of Physics \& Astronomy, Texas A\&M University-Commerce, Commerce, TX, 75429, USA
}

During the Spring 2020 semester, classes at a medium-sized regional rural university transitioned from faceto-face (FTF) to fully online instruction following Spring Break due to the COVID-19 pandemic. Learning assistants teaching in STEM disciplines experienced difficulties during this transition unique to our student population consisting of many nontraditional and first generation students living in rural areas. We conducted a phenomenological study about these LAs experiences using a Community of Practice (CoP) theory lens. Through interviews with 10 LAs, novel conversations about moving from FTF courses to a virtual setting emerged. In response to the interruptions of the CoP, LAs discussed their new learning opportunities. In this paper, we highlight the opportunities and challenges as encountered by the LAs. Considerations and concerns for instructors with similar student populations are presented. 


\section{INTRODUCTION}

Due to the pandemic, universities nationwide transitioned to online instruction in the middle of the Spring 2020 semester. Instructors transformed face-to-face (FTF) classes to purely online classes with little notice, and students who had not planned on taking an online class were thrust into this new learning environment. Amongst these instructors and students were learning assistants (LA). LAs hold a dual role of instructor for one class and students in their own classes. They can provide a unique perspective on the challenges and opportunities of online instruction.

Over 100 LA programs exist at universities nationwide. LAs are undergraduates who assist in teaching courses that they themselves have taken recently. The LA model is distinct from other models of student instructors such as Supplemental Instruction and undergraduate teaching assistants in that LAs take a pedagogy class and are engaged as part of a teaching and learning community $[1,2]$. LA programs have been found to have many benefits: improved learning for both the LAs and their students, course transformation, and faculty professional development [3-7]. Additionally, high school teachers who had been LAs were found to be more likely to use reformed teaching practices than those who had not [8].

LAs work in a variety of environments including both studio and lecture classrooms. Some work in recitation sections. In online settings, they can facilitate group work in break out rooms in video conferencing platforms such as Zoom, facilitate discussion boards, or tutor students outside of "regular" class. However, little work to-date has focused on LAs working in online learning environments $[9,10]$. The challenges faced by instructors and students depend on the context of the university and whether synchronous classes are possible. At a rural university, synchronous classes may not be possible due to the poor internet connection in many rural areas. The socioeconomic status of a university's students also determine the quality of electronic devices and internet connections students use to access online courses. In this paper, we present the experiences of LAs at a medium sized regional rural university in Texas during the transition to online instruction.

\section{THEORETICAL FRAMEWORK}

Learning occurs in situations where there is a community of practice (CoP) [11]. LAs participating in these communities can develop discipline-based and teaching identities [2]. The LAs in this study participated in an authentic social environment to learn about teaching. Membership in the LAs community occurs through regular participation in different elements of the LAs program. The LAs experienced a CoP in the following ways: During weekly preparation sessions, the course instructor collaborated with and mentored the LAs. Detailed discussions with the instructor around the course content and following week's materials provided opportunities for LAs to engage in authentic practices of teaching and learning. During the pedagogy class, LAs were asked to share their week's challenges or successes when facilitating students learning in classrooms by relating their experience to pedagogy thus reinforcing their teaching practices. Additionally, the pedagogy class brought a wide network of STEM LAs together to build relationships. The class provided opportunities for LAs to learn from one another's experiences across disciplines. Chatting about their challenges in informal social settings - such as during their class breaks in the student lounge - also reinforced the community network and promoted support.

\section{RESEARCH QUESTION}

As part of a larger research project, we conducted semistructured interviews with LAs. After discussing the LA program, their experiences and their LA identity, a final question was posed. The researcher asked if there was anything else the LA wanted to talk about or explain. This final question led to a conversation about the quarantine during COVID-19 and their unique experience as an LA during remote teaching. Therefore, the research question addressed in this paper is 'What is the experience of an LA during remote teaching and learning?' The emergent question was, "What are the opportunities and challenges an LA faces during remote teaching and learning?".

\section{METHODS}

\section{A. Data collection}

We interviewed 10 LAs at a medium-sized public regional university. Our institution is an emerging Hispanic institution that serves a rural population. A large number of nontraditional and first-generation college students make up our classes. There were 38 LAs divided amongst six subjects: math, biology, chemistry, physics, integrated science, and astronomy courses. All 38 LAs received an email to voluntarily interview about their experiences, and 10 agreed. LAs interviewed taught in four out of the six subjects: introductory physics, chemistry and math classes and an astronomy elective. The physics classes are taught in studio mode. The chemistry class is a lecture based class with a tutorial, and chemistry LAs were given their own recitation sessions. Math classes were mainly lecture based with one group work session per week. The astronomy class was lecture-based with students interacting in pairs to work on tutorials. Interviews were conducted at the end of the academic year. See Table 1 for demographic information.

Each interview was conducted virtually through Zoom due to the COVID-19 pandemic. Interviews were a minimum of thirty minutes and audio was recorded for transcription. These interviews became candid. Trust was established 
TABLE I. Participant demographics (a total of $n=10$ ).

\begin{tabular}{cc}
\hline \hline Gender & $\mathbf{n}$ \\
Male & 4 \\
Female & 6 \\
& \\
LA Class & \\
Physics & 4 \\
Math & 2 \\
Chemistry & 2 \\
Astronomy & 2 \\
\hline \hline
\end{tabular}

quickly because of their desire to discuss their particular experiences about teaching and learning during a quarantine. Much of what they discussed during the interviews had also been mentioned in the LA classes and with program instructors.

\section{B. Data analysis}

The final interview question was transcribed specifically to highlight the phenomena of being an LA during the pandemic. The researcher noticed when she asked about their overall thoughts of their experience or if they had anything to add about the LA experience, each participant chose to discuss what it was like to be an LA and student in the virtual setting. All 10 LAs demonstrated a desire to discuss the phenomena. We take a phenomenological approach to explain their stories during this time. Phenomenological research allows the researcher to learn from lived and shared experiences [12]. The LAs were clearly learning from this unique time in our society. No assumptions were made because the conversation was unexpected. Specific ideas became apparent as more participants chose to discuss what they were feeling as a result of the pandemic. Key words and phrases were aligned to generate similarities and overarching ideas. The phrases made by the participants were tallied by idea to determine how many participants made statements aligned with each theme.

\section{FINDINGS}

As part of the LA community of practice, communication between professors, LAs, and students in our classes helped determine that the majority of our students suffered from at least one of the following obstacles to effective learning. The items marked with an asterisk particularly impact the rural population with a large fraction of the non-traditional, first generation college students we serve.

- They have children of their own, and child-caring and homeschooling take up the majority of their time.*
- They are required to help out on the farm or family business.*

- They are required to care for and/or homeschool siblings/other family members.*

- No workspace at home suitable for academic study.*

- Unreliable or no internet, and are unable to access free internet plans offered by ISPs.*

- Many students already work up to 40 hours a week and now are asked to take on extra shifts.*

- Students at freshman and sophomore levels in particular are still developing time management and study skills. The decrease in structure negatively affects their ability to work efficiently.

- Serious mental health problems exacerbated by the lockdown.

Our students are almost all home with their parents or children. Many have a home environment that strongly inhibits academic study. We also learned from conversations, the parents of first generation college students could be unsympathetic to the time demands and work environment required for academic study. Although we have no direct evidence that students in the classes the LAs instructed experienced domestic abuse, it has been shown that such incidents increased during lockdown [13].These commonalities present commonalities to this specific group of LAs, also promoting a unique community of practice. They have endured and learned together through this challenging time.

In each interview, we spent between 3 and 10 minutes discussing how the LAs felt about the last few weeks of the semester. They often mentioned some of the issues listed above. Most moved home and took on additional work and were living with family. However, the main conversation was about how changing to remote learning posed specific opportunities and challenges to them. The LAs clearly had meaningful insights into teaching and learning during the pandemic. They told stories of how they felt as an LA and as a student. From their shared experiences, as part of a specific community of practice, we developed a list of opportunities and challenges faced by LAs in the remote teaching environment. Table 2 highlights the frequency of the statements based on ideas.

TABLE II. Number of participants who identified the opportunity or challenge (out of a total of $n=10$ )

\begin{tabular}{ll}
\hline Opportunities & $\mathbf{n}$ \\
Creativity in contact & 7 \\
Creativity in writing & 5 \\
& \\
Challenges & 8 \\
Engagement & 6 \\
Understanding & 8 \\
Usefulness & \\
\hline \hline
\end{tabular}




\section{A. Opportunities}

\section{Creativity in Contact}

LAs needed to be creative in finding tools to reach students. In particular, they used Remind, Google Hangout, GroupMe, Discord, and even text messages. They mentioned that they needed to spend more time with individual students, rather than large groups, to accommodate for the individual needs of students. Virtual meetings were typically more productive when one-on-one. In fact, they began to feel more important to specific individuals. One participant said:

Participant B: I feel like I can put myself out there more when I meet with the students one at a time, they like to just text too.

Another mentioned,

Participant G: We had to be flexible for different times to meet because it was just so crazy, there were not times when a whole group could meet, so we had to work it out one-onone.

These quotes demonstrate how flexible and willing the LAs were to make connections with students. Most LAs wanted their students to know they were not alone, so they provided detailed one-on-one tutoring and leveraged mobile apps. We argue that LAs play an important role in providing prompt feedback to the students in an online setting, where timely access to faculty is not always possible.

\section{Creativity in Writing}

Furthermore, LAs realized they had to learn to explain more clearly in writing. They learned to be more explicit, direct, and detailed. The LAs who mentioned these phenomena realized that it was a new skill to write such detailed instructions and a significant benefit. One LA explained,

Participant A: I didn't realize that writing would be so important in the STEM subjects, we often just discuss the content, but for us to be successful, we had to write the directions because many don't come out for the virtual meetings. They rely on the written explanations alone.

Another participant said,

Participant H: Being specific with directions is helpful to the students to navigate the course, instructions so they know what we want from them.

LAs learned to be flexible in communication and more explanatory in writing.

\section{B. Challenges}

Conversely, the LAs realized quite often that there was a challenge even when an opportunity presented itself.

\section{Engagement}

The LAs remarked that there was a lack of engagement from their students. Students were not attending the offered virtual tutorials as often as they did FTF sessions. While online attendance increased when an exam approached, the attendance was still much lower than that for FTF reviews. Also, the students were not as engaged in whole group discussion during virtual classes. The students were less likely to discuss, answer questions, or ask questions. Participant I mentioned, 'Students were not engaged and didn't come to the zoom sessions."

The LAs empathized with their students because they also lost personal engagement as students in their own classes. They lost FTF interaction and group work both in their own classes and in the classes that they taught. When they went online, LAs felt that they no longer benefited from that engagement and that they were no longer able to help their students either. One of the participants declared,

Participant A: There was a lack of dimension in the virtual settings, which I think made it less engaging.

Another participant reported,

Participant H: We couldn't interact with them personally - we wouldn't ask the students individually "do you understand"... and read their body language. Whereas in the online setting it was more like each LA was holding its own mini-lecture.

The relative level of engagement before and after the transition online depended strongly on the modality of the class before the transition online. One participant noted that LAs were able to interact more with students in the online setting, where the instructor had set up Zoom break-out rooms for small groups, than in the lecture setting where interaction was via a think-pair-share model and moving around the classroom and engaging with students was challenging.

\section{Understanding}

Both the LAs and their students struggled to understand the material through online learning in their respective classes. Each LA reported a desire to learn the material in more depth than they were able to with the asynchronous learning. One LA noted,

Participant D: I could understand where the students were coming from, learning these subjects online is hard. We don't choose these majors to be easy, we want to know the stuff, but without the professor, it's challenging.

The LAs mentioned that they did not learn as much from teaching online as they do FTF because they were not able to engage in ongoing discussion online.

\section{Usefulness}

In a variety of ways, the LAs mentioned they struggled to feel useful. Participant C commented, "I wasn't able to help, 
but it really bummed me out because I like being an LA." In particular, this participant was not able to access the online learning system. Others were not used once the class went online. Participant F described, "the professor didn't really need my help." The ten participants were asked if they felt extremely involved, moderately involved, or not involved. Out of the 10 LAs interviewed, two students felt extremely involved and six of the LAs felt moderately involved in the online portion of the semester, but much more helpful when they were FTF. The LAs who reported to be moderately involved often mentioned that the students in the class did not seek help or could not access the online sessions. In these instances, their lack of usefulness was because they would show up to help online and the students would not come to the virtual meetings. However, some of the LAs continued to feel useful to the professor. Two reported they did not feel involved at all. Several mentioned they were not confident the professors knew how to use them more. Yet, most remarked they felt like they were more of a resource, rather than the teacher.

\section{DISCUSSION AND CONCLUSION}

The CoP of LAs was disrupted by the transition to online teaching, and prompted a need for the LAs to reflect on what was gained and lost by the move. The LAs enjoyed and learned from their experiences teaching in the classroom and missed the FTF interactions with the other LAs and students in the classroom. They often commented on the value of the time spent with LAs from every discipline. The camaraderie yields discussion to improve their experiences and discuss issues and concerns. These are common features of a CoP.

Despite the disruption to the CoP and the difficulty transitioning to online teaching, all the LAs found something to learn and sought ways to remain involved. The LAs were mostly positive about their experience, with eight out of ten feeling they made contributions to the online learning environment. They expressed a strong desire to continue in their role as an LA beyond this semester, regardless of the future mode of classes.

They reported the LA pedagogy class played an important role in helping the transition. Through this class, the LAs received tools to use with remote instruction such as articles and ideas on how to maintain relationships with students who had limited virtual access and limited desire to meet in whole group sessions. Some of the articles also provided validation of the struggles the LAs experienced with the sudden transition online, and discussed issues such as the mental health impact of lockdown. LAs empathized with their students' difficulties with online classes and provided useful feedback to their professors about what aspects of the online class worked and failed to work.

Virtual chat rooms and Zoom Breakout sessions were offered for students to discuss topics. However, the LAs felt the need to revert to direct instruction in mini-lectures because they felt the available technology impeded conversation. Time restraints and limited connectivity prevented rich discussion in the virtual chat rooms and breakout rooms. While those moments provided less off-task conversation and the LAs were able to focus on the content, the students were not committed to discussion. Students simply wanted the LA to be direct about course information. We know community building and content exploration takes place during off-task FTF conversations. LAs, consciously or unconsciously, leveraged detailed writing because they could not view body language, use questioning techniques, or make use of think-pairshare strategies while online.

All of the courses were a mix of synchronous and asynchronous, but the synchronous sessions were not well attended. The LAs were encouraged to stay in contact with the students and they often provided one-on-one sessions at times that were convenient for the students of the classes, which required flexibility of the LA to use a variety technology and communication methods including ones professors do not usually use in classes such as Discord and WhatsApp. The LAs were keen to stay involved, but felt disconnected from the planning and the interactions with the course instructors. Most of the prep sessions with the professors halted when classes moved online. LAs posed these types of questions during the interviews: How can we help more?, How can we keep our role as an LA? Are we important? Is virtual teaching and learning always going to be this challenging?

LAs' reflective responses about their usefulness, engagement, and understanding when transitioning online will help LA programs across the US to ensure an authentic LA experience during online/remote instruction and be mindful of supporting the LAs as both learners and teachers during these exceptional times. Specifically, universities who have rural populations with high numbers of non-traditional and first generation students need to consider how to effectively transition to an online LA model in which LAs are trained and mentored to deal with the unique challenges faced by such students. The LA pedagogy class plays a central role in this, and needs to incorporate material relevant to online pedagogy in general and to the particular needs of the student demographics. By promoting the continuation of the CoPs for LAs in the online environment, the LAs experience growth in their learning and teaching skills that benefit students with different backgrounds (e.g., providing one-on-one tutoring to those who need assistance learning the variety of technology), and using different formats (e.g., using written representation as opposed to verbal communication). These skills would also enhance LAs teaching in FTF settings.

\section{ACKNOWLEDGMENTS}

This work is funded in part by NSF and PhysTEC. 
[1] V. Otero, P. S., and N. Finkelstein, A physics departmentâs role in preparing physics teachers: The colorado learning assistant model, American Journal of Physics 78, 1218 (2010).

[2] E. Close, J. Conn, and H. G. Close, Becoming physics people: Development of integrated physics identity through the learning assistant experience, Physical Review Physics Education Research 12, 010109 (2016).

[3] R. M. Goertzen, E. Brewe, L. H. Kramer, L. Wells, and D. Jones, Moving toward change: Institutionalizing reform through implementation of the learning assistant model and open source tutorials, Physical Review Physics Education Research 7, 020105 (2011).

[4] S. J. Pollock and N. D. Finkelstein, Sustaining educational reforms in introductory physics, Physical Review Physics Education Research 4, 010110 (2008).

[5] J. White, B. Van Dusen, and E. Roualdes, The impacts of learning assistants on student learning of physics, edited by in 2016 Physics Education Research Conference ProceedingsD. L. Jones, L. Ding, and A. Traxler.

[6] N. McHenry, A. Martin, A. Castaldo, and D. Ziegenfuss, Learning assistants program: Faculty development for conceptual change, International Journal of Teaching and Learning in Higher Education 22, 258 (2010).
[7] D. Caravez, J. De La Torre, A. amd Nissen, and B. Van Dusen, Longitudinal associations between learning assistants and instructor effectiveness, edited by in 2017 Physics Education Research Conference ProceedingsL. Ding, A. Traxler, and Y. Cao.

[8] K. E. Gray, D. C. Webb, and V. K. Otero, Effects of the learning assistant model on teacher practice, Physical Review Physics Education Research 12, 020126 (2016).

[9] J. Weidler-Lewis, D. Pierce, and C. Walter.

[10] S. B. Wilson and P. Varma-Nelson, Characterization of firstsemester organic chemistry peer-led team learning and cyber peer-led team learning studentsâ use and explanation of electron-pushing formalism, Journal of Chemical Education 96, 25 (2019).

[11] J. Lave and E. Wenger, Situated learning: Legitimate peripheral participation (Cambridge University Press, 1991).

[12] E. Husserl, The crisis of European sciences and transcendental phenomenology: An introduction to phenomenological philosophy (Northwestern University Press, 1970).

[13] K. Usher, N. Bhullar, J. Durkin, N. Gyamfi, and D. Jackson, Family violence and covid-19: Increased vulnerability and reduced options for support, International journal of mental health nursing (2020). 